\title{
Nachhaltige kommunale Haushaltssicherung durch die Koproduktion öffentlicher Wirkungen mit Bürgerinnen und Bürgern?
}

\section{Substitutive und additive Formen von Koproduktion im Vergleich}

\author{
Elke Löffler/Peter Timm-Arnold
}

Wenn es in Deutschland um die Rolle des Bürgers bzw. der Bürgerin in der öffentlichen Verwaltung geht, wird sofort nach den Kosten von Bürgerbeteiligung gefragt, sehr selten aber, was dabei an Ergebnissen herauskommt, seien es Wirkungsverbesserungen für die Verwaltungsadressaten oder Sparbeiträge für den Haushalt. In diesem Beitrag richtet sich der Blick auf die Rolle von Bürgerinnen und Bürgern als Koproduzenten öffentlicher Leistungen und Wirkungen. D.h. es geht nicht nur um Mitsprache, sondern um Mitmachen. Der Beitrag zeigt neue Strategien zur Haushaltssicherung durch eine ressourcenorientierte Perspektive von Koproduktion auf. Insbesondere werden anhand von Szenarien substitutiver und additiver Koproduktion mit illustrativen Beispielen die Auswirkungen von Koproduktion auf Einsparungen bei der kommunalen Leistungserbringung, die Qualität öffentlicher Leistungen und öffentliche Wirkungen kritisch diskutiert. Dabei wird deutlich, dass effektive Koproduktion nicht heißt, Bürgerinnen und Bürger als Lückenbüßer für eingesparte öffentliche Leistungen zu missbrauchen. Anschließend illustriert eine Fallstudie aus der niederländischen Gemeinde Zeist, wie in einem innovativen sparpolitischen Ansatz des Spardialogs mit Bürgerinnen und Bürgern, deren Expertise und Kreativität, eine Haushaltslücke von sechs Millionen Euro mit konstruktiven Sparvorschlägen geschlossen werden konnte.

\section{Kosten und Nutzen der Zusam- menarbeit mit Bürgern aus kom- munaler Sicht}

Die Zusammenarbeit zwischen Kommunen und Bürgerinnen und Bürgern wird in

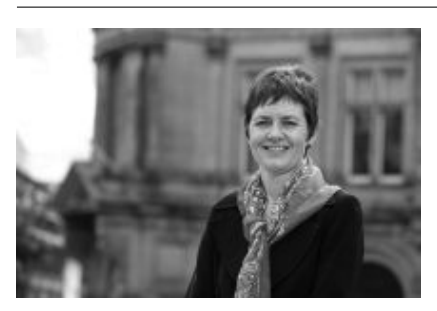

Dr. Elke Löffler

Verwaltungswissenschaftlerin, Geschäftsführerin Governance International
Deutschland noch meist unter den Begriffen Bürgerbeteiligung bzw. Bürgerkommune subsumiert. Dabei wird bei knappen Budgetmitteln schnell die Frage nach den Kosten verschiedener Verfahren gestellt,

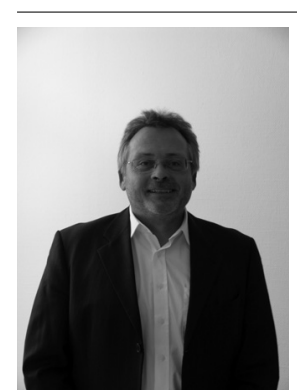

Dr. Peter TimmArnold

Politikwissenschaftler, Partner Governance International aber nur selten ex-ante gewünschte Wirkungsverbesserungen definiert. Insofern ist es schwierig, den Erfolg von Bürgerbeteiligungsverfahren zu beurteilen. Als einzige Kennzahl wird oftmals auf die Zahl der teilnehmenden Bürgerinnen und Bürger abgehoben, die jedoch wenig darüber aussagt, welchen Nutzen die Kommune und beteiligte Bürgerinnen und Bürger durch ihren Dialog gewonnen haben.

In diesem Beitrag richtet sich der Blick auf die Rolle von Bürgerinnen und Bürgern als Koproduzenten öffentlicher Leistungen und Wirkungen. D.h. es geht nicht nur um Mitsprache, sondern auch um Mitmachen. Mit Koproduktion werden wirkungsorientierte Formen des gemeinsamen Handelns zwischen öffentlichen Verwaltungen und Bürgerinnen und Bürger bezeichnet, die darauf angelegt sind, die Fähigkeiten, Potenziale und Beiträge aller Beteiligten besser zur Entfaltung zu bringen, um die Lebensqualität zu verbessern oder Effizienzgewinne zu erzielen. ${ }^{1}$

Dabei geht es sowohl um die Zusammenarbeit von Kommunen mit ehrenamtlich engagierten Bürgerinnen und Bürgern (z.B. im Rahmen von Patenmodellen), als auch Verhaltensänderungen von Nutzerinnen und Nutzern durch präventive Maßnahmen (z.B. Gesundheitsvorsorge durch mehr Bewegung) bis $\mathrm{zu}$ neuen digitalen Formen von Lebenshilfen (z.B. E-Health und E-Care Lösungen).

Vgl. Löffler 2015, S. 319. 


\begin{tabular}{|c|c|c|c|}
\hline \multirow{4}{*}{$\begin{array}{l}\text { Beteiligung von } \\
\text { Fachkräften (der } \\
\text { öffentlichen Ver- } \\
\text { waltung bzw. freier } \\
\text { Träger) }\end{array}$} & \multicolumn{3}{|c|}{$\begin{array}{l}\text { Beteiligung von Bürgern/innen (als Nutzer einer kommunalen } \\
\text { Leistung, Einwohner oder Bürgergruppe) }\end{array}$} \\
\hline & & Aktiv & Passiv \\
\hline & Aktiv & A Koproduktion & $\begin{array}{l}\text { B Dienstleistungs- } \\
\text { kommune }\end{array}$ \\
\hline & Passiv & $\begin{array}{l}\text { C Selbsthilfe, Ehren- } \\
\text { amt und Selbstorga- } \\
\text { nisation (z.B. Vereins- } \\
\text { tätigkeit) }\end{array}$ & D Symbolpolitik \\
\hline
\end{tabular}

Tab. 1: Begriffsklärung von Koproduktion und Abgrenzung vom Ehrenamt (Quelle: Löffler (2016) (überarbeitete Fassung))

Koproduktion bedeutet vor allem eine ermöglichende Verwaltung, die Hilfe zur Selbsthilfe und Verhaltensänderungen leistet. Insbesondere geht es darum, die Fähigkeiten von Nutzerinnen und Nutzern $\mathrm{zu}$ entfalten und $\mathrm{zu}$ heben, um diese in die Lage zu versetzen, ihre Lebensqualität und die Lebensqualität anderer Menschen $\mathrm{zu}$ verbessern. Koproduktion bedeutet daher nicht unbedingt, dass bezahlte Arbeit teilweise durch unbezahlte ehrenamtliche Tätigkeiten substituiert wird (sog. substitutive Koproduktion), sondern dass der öffentliche Sektor einen Beitrag leistet, um die Wirkungen der Beiträge von Bürgerinnen und Bürgern zu verbessern (sog. additive Koproduktion). Wenn etwa die Kommune East Dunbartonshire in Schottland Menschen mit Demenz dabei unterstützt, Newsletter für andere Menschen mit Demenz und deren Angehörige zu schreiben, erhält die Community passgerechte Informationen und die Newsletter-Autorinnen und Autoren erfahren eine wichtige Wertschätzung, die deren Lebensqualität verbessert ${ }^{2}$.

Abgesehen davon, dass das Ausmaß an Koproduktion von Verwaltungsmitarbeiterinnen und -mitarbeitern oftmals unterschätzt wird, herrscht in der öffentlichen Verwaltung in der Regel recht große Skepsis darüber, inwieweit Bürgerinnen und Bürger als Koproduzenten eine wichtige Rollen spielen können, um den öffentlichen Haushalt zu entlasten. So zeigte eine Meinungsabfrage im Rahmen von elf Fokusgruppensitzungen, die 2014 im Auftrag der Bertelsmann Stiftung in vier Bundesländern durchgeführt wurde, dass die überwiegende Mehrheit der Fokusgruppenteilnehmenden aus der öffentlichen Verwaltung für den kommunalen Haushalt keine kurzfristige Entlastung erwartete, wenn Bürgerinnen und Bürger als Koproduzenten eine wichtigere Rolle spielen. ${ }^{3}$

Im Folgenden sollen mögliche Auswirkungen von additiver und substitutiver Formen von Koproduktion auf die kommunale Haushaltssicherung anhand von Szenarien verglichen werden. Dabei wird auf die Aspekte kommunale Einsparungen, Leistungsqualität und öffentliche Wirkungen abgehoben. Damit wird freilich nur die kommunale Seite von Koproduktion abgebildet. In einem vollständigen Kosten-Nutzen-Modell müssen auch Kosten und Nutzen von Koproduktion aus Bürgersicht abgebildet werden.

\section{Begriffsklärung von Koproduktion und Abgrenzung von Ehrenamt und Bürgerbeteiligung}

Die Koproduktion öffentlicher Leistungen und Wirkungen mit Bürgerinnen und Bürgern auf kommunaler Ebene ist nichts Neues, wenngleich der Begriff und das Konzept in Deutschland noch weitgehend unbekannt und ungebräuchlich sind. Neu ist hingegen die wirkungsorientierte Umsetzung von Mit-Steuern, Mit-Entwickeln, Mit-Umsetzen und Mit-Bewerten zur Lösung alter und neuer Herausforderungen wie Arbeitslosigkeit, Umweltschutz, Energiewende, Integration und demografischer Wandel.

Koproduktion kann in unterschiedlichen Phasen öffentlicher Entscheidungsund Leistungsprozesse stattfinden. Nach dem Koproduktionsmodell von Governance International kommen Bürgerinnen und Bürgern als Nutzerin und Nutzer einer kommunalen Leistung, Einwohnerin oder Einwohner oder Bürgergruppe un- terschiedliche Rollen ${ }^{4}$ bei der Koproduktion öffentlicher Leistungen und Wirkungen zu:

- Mit-Steuern bei der Bestimmung von Prioritäten: Bürgerinnen und Bürger kennen ihre Bedürfnisse selbst am besten, was bei der Bestimmung von Wirkungszielen und beim Einsatz von kommunalen und bürgerschaftlichen Ressourcen unerlässlich ist.

- Mit-Entwickeln von Lösungen: Bürgerinnen und Bürger haben kreative Ideen, von denen die Verwaltung nichts weiß, und Wissen als , user experts".

- Mit-Umsetzen von öffentlichen Wirkungen: Bürgerinnen und Bürger bringen Fähigkeiten, Energie und Ressourcen ein, um ihre Lebensqualität und die Lebensqualität anderer Menschen zu verbessern.

- Mit-Bewerten der Wirkung: Bürgerinnen und Bürger können oft besser als die Verwaltung beurteilen, ob ein Lösungsansatz wirklich die gewünschte Wirkung erzielt hat.

Tabelle 1 verdeutlicht, worum es bei Koproduktion geht und wie Koproduktion von Formen des klassischen Ehrenamts abzugrenzen ist. Mit Koproduktion bezeichnet man, wie bereits erwähnt, vor allem Formen des wirkungsorientierten Zusammenwirkens zwischen öffentlicher Verwaltung und der Bürgerschaft, bei der sowohl Fachkräfte als auch Bürgerinnen und Bürger bzw. Nutzerinnen und Nutzer verschiedene, aber gleichermaßen wichtige Beiträge einbringen (Feld A). Weitaus verbreiteter ist jedoch das Szenario, dass Fachkräfte Leistungen für weitgehend passive oder passiv behandelte Adressaten erbringen, wie dies etwa in vielen Pflegeheimen der Fall ist (Feld B). Ebenfalls

2 Vgl. Brown/Löffler/Christie 2016.

3 Bei den vier Fokusgruppen, die das Thema Koproduktion im Handlungsfeld Junge Menschen und Familien diskutierten, waren 66 Prozent der Meinung, dass von Koproduktion kurzfristig keine Haushaltsentlastungen zu erwarten sind. Bei den drei Fokusgruppen aus dem Bereich Öffentliche Sicherheit vertraten 65 Prozent die gleiche Meinung. Bei den vier Fokusgruppen aus dem Bereich Gesundheit und Soziales vertraten sogar 80 Prozent diese Meinung. Vgl. Löffler et al. 2015, S. 19,24 und 29.

4 Vgl. Timm-Arnold/Löffler 2013. 


\begin{tabular}{|l|c|c|c|}
\hline $\begin{array}{r}\text { Formen der Zusammenarbeit } \\
\text { zwischen öffentlichen } \\
\text { Verwaltung und Bürgern }\end{array}$ & Konsultation & $\begin{array}{l}\text { Bürgerbeteiligung (Partizipation in } \\
\text { öffentlichen Entscheidungen) }\end{array}$ & $\begin{array}{l}\text { Koproduktion von öffentlichen } \\
\text { Leistungen und Wirkungen }\end{array}$ \\
\hline Richtung & beidseitig & beidseitig & öffentlicher Raum \\
\hline Raum & Öffentlicher Raum & $\begin{array}{c}\text { Öffentlicher und nicht-öffentlicher } \\
\text { Raum }\end{array}$ \\
\hline $\begin{array}{l}\text { Aktivität der öffentlichen } \\
\text { Verwaltung }\end{array}$ & $\begin{array}{c}\text { Zuhören und } \\
\text { Rückmeldung }\end{array}$ & Dialog & $\begin{array}{c}\text { Gemeinsames Handeln } \\
\text { (Mitsteuern, Mitentwickeln, } \\
\text { Mitumsetzen, Mitbewerten) }\end{array}$ \\
\hline Input seitens der Bürger & niedrig & mittel & hoch \\
\hline
\end{tabular}

Tab. 2: Die Unterschiede zwischen Konsultation, Bürgerbeteiligung und Koproduktion (Quelle: Löffler und Martin (2015), S. 303 (überarbeitete Fassung))

weit verbreitet sind Formen der Selbsthilfe (z.B. Menschen mit chronischen Erkrankungen) bzw. Formen des klassischen ehrenamtlichen Engagements für andere Menschen (z.B. pflegende Angehörige) bzw. gesellschaftliche Gruppen, oft in Vereinen organisiert (Feld C). Feld D schließlich kennzeichnet Situationen, in denen weder die öffentliche Hand noch betroffene Bürgerinnen und Bürger viel zur Problemlösung beitragen, wie dies etwa bei Symbolpolitik im Umgang mit marginalisierten Bevölkerungsgruppen oder dem Klimaschutz der Fall ist.

Damit wird auch deutlich, was Koproduktion nicht ist:

- reine Selbsthilfe von Bürgerinnen und Bürgern ohne Begleitung der öffentlichen Verwaltung

- ehrenamtliches Engagement ohne Koordination, Steuerung, Unterstützung oder sonstige Form der Beeinflussung der öffentlichen Verwaltung, wie dies etwa bei vielen Formen der informellen Hilfe etwa auf Nachbarschaftsebene der Fall ist

- Selbstorganisation von Bürgerinnen und Bürgern in Form von Vereinen, Bürgerinitiativen etc.

Umgekehrt zeigt die Vielzahl von Patenmodellen wie beispielsweise Integrationslotsenmodelle für Migrantinnen und Migranten und andere Formen des ehrenamtlichen Engagements in Zusammenarbeit mit Kommunen und Freien Trägern, dass ehrenamtliches Mit-Umsetzen sowohl im öffentlichen als auch nicht-öffentlichen Raum ein wichtiger Bestandteil von Koproduktion öffentlicher Leistungen und Wirkungen ist. Gleichzeitig umfasst Koproduktion auch andere Formen des MitUmsetzens durch Verhaltensänderung von
Nutzerinnen und Nutzern sowie Formen der Zusammenarbeit durch Mit-Steuern, Mit-Entwickeln und Mit-Bewerten von Wirkungsverbesserungen.

Insofern verlangt Koproduktion einen höheren Input von Bürgerinnen und Bürgern als Bürgerbeteiligung oder gar Konsultation, wie Tabelle 2 verdeutlicht. Bei Koproduktion ist auch die öffentliche Verwaltung gefordert und kann sich nicht auf eine Politik des Zuhörens beschränken (im Fall von Konsultation) bzw. lediglich öffentliche Dialoge (Bürgerbeteiligung) durchführen, was oftmals durch die Einrichtung von Internetplattformen geschieht. Koproduktion erfordert immer ein gemeinsames Handeln von öffentlicher Verwaltung und Bürgerinnen und Bürgern, um öffentliche Leistungen und Wirkungen zu verbessern. Dies kann durch unterschiedliche Formen von MitSteuern, Mit-Entwickeln, Mit-Umsetzen und Mit-Bewerten im öffentlichen und nicht-öffentlichen Raum erfolgen. Offensichtlich sind die Abgrenzungen zwischen den Engagementformen fließend. Des Weiteren geht es nicht um die Frage „entweder - oder“, sondern um den richtigen Mix von Engagementformen.

Dies hat auch Implikationen für den Ressourcenbedarf öffentlicher Verwaltungen. Im Folgenden sollen die Kosten und Nutzen von Koproduktion aus kommunaler Sicht weiter vertieft werden

\section{Koproduktion als Alternative zur traditionellen Haushalts- konsolidierung? Eine Bestands- aufnahme in Nordrhein-Westfalen}

In Deutschland sind viele Kommunalhaushalte - insbesondere in Nordrhein-
Westfalen (NRW) - finanziell in eine teilweise dramatische Schieflage geraten. In Einzelfällen steht wegen Überschuldung die politische Handlungsfähigkeit auf dem Spiel; in zwei Fällen in NRW ${ }^{5}$ wurde sogar ein Sparkommissar durch die Kommunalaufsicht eingesetzt, was nichts anderes ist als die temporäre Aussetzung der kommunalen Selbstverwaltung. In diesem Kontext steht vielerorts das Thema Haushaltskonsolidierung ganz oben auf der Agenda. Die Frage liegt nahe, ob der massive Sparzwang zu einem „Umdenken“ von Politik und Verwaltung in Richtung bürgerschaftlicher Koproduktion führt.

Eine aktuelle Analyse (2016) der Haushaltssanierungspläne von rund 60 Kommunen in NRW macht allerdings deutlich, dass immer noch die „klassischen“ Handlungskonzepte wie Steuererhöhungen (Grundsteuer und Gewerbesteuer), Standardreduzierungen im Pflichtaufgabenbereich und die Streichung freiwilliger Leistungen dominieren ${ }^{6}$. Die Zusammenarbeit mit der Bürgerschaft wird kaum als ein strategischer Ansatz zur Verbesserung öffentlicher Wirkungen bzw. Effizienzsteigerung eingesetzt. Insofern dominieren dialogische Formen der Bürgerbeteiligung bzw. kleinräumige Koproduktionsprojekte im Bereich freiwilliger Leistungen. So zeigt eine Auswertung ${ }^{7}$ von mehr als 3.000 Sparmaßnahmen aus 61 Kommunen im Rahmen des so genannten nordrhein-westfälischen Stärkungspaktes

Gemeinde Nideggen/Eifel und Stadt Altena/ Märkischer Kreis.

6 Vgl. Timm-Arnold/Löffler 2013.

7 Verwendete Suchbegriffe: ehrenamtlich, Ehrenamt, bürgerschaftlich, Koproduktion 
Stadtfinanzen $^{8}$, dass sich lediglich vier (!) dem hier interessierenden Themenfeld zuordnen lassen:

- Verstärkter Einsatz von Ehrenamtlichen in der Bücherei

- Stellenabbau durch ehrenamtliches Engagement

- Reduzierung des Zuschussbedarfes für ein Freibad durch zusätzliches bürgerschaftliches Engagement

- Durchführung der Museumsaufsicht durch Ehrenamtliche

Man sieht überdeutlich: Nachhaltige Haushaltssicherung durch Wirkungsverbesserung ist in deutschen Kommunen noch nicht angekommen. Im Gegenteil: Die hier genannten Maßnahmen lassen sich eher der Kategorie „Lückenbüßer“ (siehe Szenario 1 unten in Tabelle 3) für fehlende öffentliche Ressourcen oder verfehlte Politik zuordnen, da unklar ist, inwiefern sich durch den verstärkten bürgerschaftlichen Einsatz die kommunalen Leistungen oder gar Wirkungen kommunalen Handels verbessert haben. ${ }^{9}$ Das ist

\begin{tabular}{|l|l|l|}
\hline $\begin{array}{l}\text { Form von Koproduktion } \\
\text { (aus ressourcenorientierter } \\
\text { Sicht) }\end{array}$ & $\begin{array}{l}\text { Ressourcen der } \\
\text { öffentlichen Verwaltung } \\
\text { (insb. Haushalts- und } \\
\text { Personalmittel) }\end{array}$ & $\begin{array}{l}\text { Ressourcen der } \\
\text { Bürgerinnen und Bürger } \\
\text { (insb. Zeit und finanzielle } \\
\text { Mittel) }\end{array}$ \\
\hline Substitutiv & Abnahme & Zunahme \\
\hline Additiv & Zunahme & Zunahme oder Abnahme \\
\hline
\end{tabular}

Tab. 3: Additive und substitutive Formen von Koproduktion (Quelle: Löffler 2016 (überarbeitete Fassung))

kungsorientierte Koproduktionsstrategie zu entwickeln, die innovativen Nischenprojekten den Weg in den Mainstream eröffnet, um Wirkungsverbesserungen zu erzielen.

\section{Ein ressourcenorientierter Ansatz von Koproduktion}

\section{Additive und substitutive Formen der Koproduktion}

Koproduktion wird oftmals kritisch auf eine kommunale Einsparstrategie reduziert, bei der es darum geht, kommunale

\section{"Der Koproduktionsansatz ist in deutschen Kommunen noch entwicklungsfähig. Dabei gilt es, auf bestehende Koproduktionsformen aufzusetzen und eine wirkungsorientierte Koproduktionsstrategie zu entwickeln."}

nicht ungefährlich, denn „... wenn der Bürger sich zum Lückenbüßer degradiert fühlt, der nun umsonst den Job der Verwaltung erledigen soll, (...) besteht die Gefahr, dass er sich dann andere interessantere Freizeitaktivitäten suchen wird ". ${ }^{10}$ Viele, mit viel Engagement und „Herzblut" gestartete Aktivitäten sind aus diesem Grund am Ende an der kommunalen Wirklichkeit gescheitert.

Insofern ist der Koproduktionsansatz in deutschen Kommunen noch entwicklungsfähig. Dabei gilt es, durch eine Bestandsaufnahme auf bestehenden Koproduktionsformen aufzusetzen und eine wir-
Ressourcen (vor allem Haushaltsmittel und Personal) durch für die Kommune kostenlose Ressourcen der Zivilgesellschaft in Form von ehrenamtlicher Arbeit zu ersetzen. Diese Form von Koproduktion wird als substitutive Koproduktion bezeichnet $^{11}$. So zeigt die Bestandsaufnahme in Nordrhein-Westfalen, dass es in diesem Fall darum geht, mehr (für die Kommune) kostenlose Ressourcen der Bürger/innen zu erschließen, um das notwendige Leistungsangebot zu gewährleisten.

Umgekehrt kann Koproduktion auch bedeuten, dass sich Kommunen in Bereichen engagieren, in denen Bürgerinnen und Bürger sich bisher selbst organisiert hatten (vgl. Feld C in Tabelle 1) bzw. sich weder die öffentliche Hand noch die Bürgerschaft gekümmert haben (Feld D in Tabelle 1). Auch dieser Trend ist in Nordrhein-Westfalen zu identifizieren: Beispielsweise stärkt die „Quartiersakademie Nordrhein-Westfalen“ seit Jahresbeginn 2016 zivilgesellschaftlich im Quartier engagierte Menschen und Initiativen und qualifiziert sie bei der Gestaltung ihrer Quartiere zum Akteur und Mitgestalter in ihrer Kommune.

Der verstärkte Ressourceneinsatz von Kommunen kann dazu dienen, Hilfe zur Selbsthilfe bzw. Crowdfunding zu stärken. Bei dieser Form der additiven Koproduktion werden zumindest mittelfristig auch die Potenziale von Bürgerinnen und Bürgern gehoben, so dass sie verstärkt Ressourcen einbringen. In anderen Fällen der additiven Koproduktion kann das verstärkte Engagement von Kommunen eingesetzt werden, um schon (zu) stark ehrenamtlich engagierte Bürgerinnen und Bürger, wie pflegende Angehörige, zu entlasten. Additive Koproduktion bedeutet in jedem Fall einen verstärkten Ressourceneinsatz der Kommune.

Bei Kommunen, die unter Sparzwängen stehen, insbesondere Haushaltssicherungskommunen in NRW, ist es einfacher, politischen Konsens für substitutive For-

8 http://gpanrw.de/media/1435306026_staerkungspakt_stadtfinanzen_-_manahmenkatalog_2015.pdf (Abruf: 05.06.2016).

9 Natürlich ist gegen den Einsatz Ehrenamtlicher in der Bücherei nichts einzuwenden, wenn dadurch mehr Menschen die Bibliothek nutzen, die sonst nicht in die Bücherei gehen oder die Schwelle für Nutzer (z.B. Kinder und Jugendliche) herabgesetzt wird.

10 Vgl. Bogumil/Holtkamp 2010, S. 397.

11 Vgl. Löffler/Watt 2009. 


\begin{tabular}{|l|c|c|c|c|c|}
\hline Szenarien & $\begin{array}{l}\text { Ressourcen der } \\
\text { öffentlichen } \\
\text { Verwaltung }\end{array}$ & $\begin{array}{l}\text { Ressourcen der } \\
\text { Bürgerinnen } \\
\text { und Bürger }\end{array}$ & $\begin{array}{l}\text { Einsparungen bei } \\
\text { der öffentlichen } \\
\text { Leistungserbringung }\end{array}$ & $\begin{array}{l}\text { Qualität } \\
\text { öffentlicher } \\
\text { Leistungen }\end{array}$ & $\begin{array}{l}\text { Verbesserung } \\
\text { öffentlicher } \\
\text { Wirkungen }\end{array}$ \\
\hline $\begin{array}{l}\text { 1.,,Bürger als } \\
\text { Lückenbüßer“ }\end{array}$ & - & + & $\begin{array}{c}+ \\
\text { (kurzfristig) } \\
-\end{array}$ & - & - \\
\hline $\begin{array}{l}\text { 2.,„Billiger, aber } \\
\text { nicht besser“ }\end{array}$ & - & + & + & $?$ & $?$ \\
\hline $\begin{array}{l}\text { 3. „Billiger und } \\
\text { besser“ }\end{array}$ & - & + & + & $?$ & $?$ \\
\hline
\end{tabular}

Tab. 4: Haushaltseffekte substitutiver Koproduktionsformen (Quelle: Löffler (2016) (überarbeite Fassung))

men als für additive Formen von Koproduktion zu finden. Mit dem einseitigen Fokus auf den kurzfristigen Ressourcenaufwand der Kommune geraten jedoch oftmals die Ergebnisse von substitutiven und additiven Formen von Koproduktion aus dem Blickfeld. Gerade unter Haushaltssicherungsgesichtspunkten sollte jedoch evaluiert werden, ob die jeweilige Koproduktionsform auch mittelfristig zu kommunalen Einsparungen führt bzw. die Qualität kommunaler Leistungen und/ oder öffentliche Wirkungen verbessert.

\section{Haushaltseffekte substitutiver Koproduktionsformen}

Im Folgenden sollen modellhaft jeweils drei besonders relevante Szenarien (vgl. Tab. 4) von substitutiver Koproduktion unter dem Aspekt kommunaler Haushaltssicherung anhand eines illustrativen Beispiels erörtert werden.

In Szenario 1 aktiviert die öffentliche Verwaltung Bürgerinnen und Bürger als Ersatz für geringer (und unzureichender) werdende Unterstützung freiwilliger kommunaler Aufgaben. Im Falle eines vollständigen öffentlichen Leistungsabbaus handelt es sich im Extremfall um keine Koproduktion mehr.

Die von Freiwilligen betriebenen Stadtteilbibliotheken stellen eine solche extreme Form substitutiver Leistungserbringung in der Kommune dar. Aufgrund des Sparzwanges werden nicht selten mit Verwaltungspersonal betriebene Zweigstellen geschlossen. Stattdessen werden die Zweigstellen durch engagierte Bürger „notdürftig“ weiter betrieben, um z.B. in sozial belasteten Stadtteilen ein Minimum an Bildungseinrichtung aufrecht zu erhalten.
Wenn Bürgerinnen und Bürger als Lückenbüßer Aufgaben übernehmen, denen sie nicht gewachsen sind bzw. die sie nicht gerne übernehmen wollen, schwinden kurzfristige Einspareffekte mittelfristig, da das bürgerschaftliche Engagement schnell nachlassen wird, so dass der bisherige Umfang des Leistungsangebots nicht aufrechterhalten werden kann. Des Weite- führen, dass die Nutzerinnen und Nutzer dieser Leistungen auch Einbußen ihrer Lebensqualität hinnehmen müssen. Ein Beispiel ist die Einstellung der kostenlosen regelmäßigen Abholung von Gartenabfällen durch die Kommune. Den Bürgerinnen und Bürgern bleibt die Wahl, Gartenabfälle entweder selbst zur Mülldeponie bzw. Häckselplatz zu bringen bzw. eine Gebühr für die regelmäßige Abholung zu bezahlen. Dadurch spart die Kommune zwar Haushaltsmittel ein, aber aus Sicht der Gartenbesitzer verschlechtert sich das kommunale Leistungsangebot. Insbesondere werden Verhaltensweisen gefördert, kleine Mengen

\section{"Wenn Bürgerinnen und Bürger als Lückenbüßer Aufgaben übernehmen, denen sie nicht gewachsen sind bzw. die sie nicht gerne übernehmen wollen, schwinden kurzfristige Einspareffekte mittelfristig, da das bürgerschaftliche Engagement schnell nachlassen wird."}

ren müssen bezahlte Fachkräfte u.U. Zeit dafür verwenden, die Fehler der unzureichend begleiteten ehrenamtlichen Kräfte wettzumachen, wodurch die Einsparungen bei der Leistungserbringung weiter abnehmen. Die Qualität des Leistungsangebots verschlechtert sich ebenfalls und führt bei Nutzern und Nutzerinnen zu einer Verschlechterung der Lebensqualität.

In Szenario 2 ersetzt die Kommune eigene Ressourcen teilweise durch Beiträge von Bürgerinnen und Bürgern bzw. Nutzerinnen und Nutzern kommunaler Leistungen, wodurch zwar Einsparungen bei der öffentlichen Leistungserbringung erzielt werden, die Qualität dieser Leistungen aber gleich bleibt oder sich möglicherweise sogar verschlechtert. Dies kann dazu von Gartenabfällen im Haushaltsmüll zu verstecken bzw. Gartenmüll „in der Natur" zu entsorgen.

In Szenario 3 handelt es sich ebenfalls um eine substitutive Form der Koproduktion, wobei in diesem Fall Kommunen durch die Beiträge von Bürgerinnen und Bürgern bzw. Nutzerinnen und Nutzern zur öffentlichen Leistungserstellung neue Kompetenzen erhalten, so dass sich in diesem „Billiger und Besser“-Szenario die Qualität öffentlicher Leistungen verbessert. Dadurch kann sich auch die Lebensqualität von Nutzerinnen und Nutzern dieser Leistungen verbessern. Die Stadt Arnsberg (NRW) arbeitet im Rahmen der Initiative "Neue Nachbarn Arnsberg"12

12 Vgl. Vogel 2016. 


\begin{tabular}{|c|c|c|c|c|c|}
\hline Szenarien & \begin{tabular}{|l|} 
Ressourcen der \\
öffentlichen \\
Verwaltung \\
\end{tabular} & \begin{tabular}{|l|} 
Ressourcen der \\
Bürgerinnen und \\
Bürger \\
\end{tabular} & \begin{tabular}{|l} 
Einsparungen bei \\
der öffentlichen \\
Leistungserbringung \\
\end{tabular} & \begin{tabular}{|l|} 
Qualität \\
öffentlicher \\
Leistungen \\
\end{tabular} & $\begin{array}{l}\text { Verbesserung } \\
\text { öffentlicher } \\
\text { Wirkungen } \\
\end{array}$ \\
\hline $\begin{array}{l}\text { 4. Freiwillige Leistungen } \\
\text { ohne erkennbare } \\
\text { Ergebnisse }\end{array}$ & + & + & - & - & - \\
\hline $\begin{array}{l}\text { 5. Bessere Leistungen durch } \\
\text { Synergien }\end{array}$ & + & + & - & + & + \\
\hline $\begin{array}{l}\text { 6. Nachhaltige Wirkungs- } \\
\text { verbesserungen durch } \\
\text { Verhaltensänderungen }\end{array}$ & $\begin{array}{c}+ \\
\text { kurzfristig } \\
- \\
\text { mittelfristig }\end{array}$ & $\begin{array}{c}+ \\
\text { kurzfristig } \\
- \\
\text { mittelfristig }\end{array}$ & $\begin{array}{c}\text { - } \\
\text { kurzfristig } \\
+ \\
\text { mittelfristig }\end{array}$ & + & + \\
\hline
\end{tabular}

Tab. 5: Haushaltseffekte additiver Koproduktionsformen (Quelle: Löffler (2016) (überarbeitete Fassung))

mit Geflüchteten zusammen, um deren Lebensqualität und das gesellschaftliche Miteinander in Arnsberg zu verbessern. Beispielsweise engagieren sich einige Geflüchtete als ehrenamtliche Übersetzerinnen und Übersetzer. Dadurch spart die Stadt Arnsberg die Honorare für professionelle Übersetzungsbüros ein und kann diese Mittel für weitere Sprach- und Förderangebote einsetzen. Zugleich wird auch die Leistungsqualität verbessert, da die ehrenamtlichen Kräfte sprachlich sensibilisiert und besser in der Lage sind, „den richtigen Ton“ zu treffen. Arnsberg sorgt durch die Bereitstellung eines Büros mit entsprechenden PCs (mit arabischen Tastaturen) für gute Arbeitsbedingungen. Das Ehrenamt wird durch eine Zusammenarbeit auf Augenhöhe mit dem Bürgermeisteramt und anderen Dienststellen wertgeschätzt. Für die als Übersetzer tätigen Geflüchteten ist es ein erster Schritt zur Integration und eine Verbesserung ihrer Lebensqualität. Durch die Verbesserung der Kommunikation der Stadt Arnsberg wiederum erhöht sich die Lebensqualität weiterer Geflüchteter.

\section{Haushaltseffekte additiver Koproduktionsformen}

Im Falle von additiver Koproduktion entstehen positive Haushaltssicherungseffekte vor allem durch Wirkungsverbesserungen. Im Folgenden sollen wieder drei Szenarien (vgl. Tab. 5) mit einem Fallbeispiel erläutert werden.

In Szenario 4 ergänzt die Kommune die Beiträge von Bürgerinnen und Bürgern durch (gut gemeinte) freiwillige Leistungen, die aber an den Bedürfnissen der
Zielgruppe vorbeigehen bzw. in Konkurrenz zu bestehenden Angeboten ehrenamtlicher Kräfte stehen, ohne diese sinnvoll zu ergänzen. In diesem Fall steigt der Ressourcenaufwand der Kommune an, ohne dass sich das kommunale Leistungsangebot oder die Lebensqualität der Betroffenen insgesamt verbessern. Beispielsweise hat der Landkreis Surrey in Großbritannien vor Beginn des Change-ManagementProzesses 2009 im Bereich Jugendarbeit zahlreiche Konsultationen mit Jugendlichen durchgeführt. Die Befragungen zeigten, dass es nicht an Angeboten für Jugendliche fehlte, sondern viele Angebote der Kommune und ehrenamtlicher Kräfte mangelhaft genutzt wurden ${ }^{13}$. Insofern verharrte die Zahl der Jugendlichen ohne Schulausbildung, Beschäftigung oder Ausbildung (NEET = „Not in Education, Employment or Training“) zwischen 2003 und $2012 \mathrm{zu}$ jedem gegebenen Zeitpunkt bei ca. $1.000^{14}$.

In Szenario 5 investieren sowohl die Kommune und engagierte Bürgerinnen und Bürger mehr Ressourcen, um das Leistungsangebot in der Kommune und die Lebensqualität gesellschaftlicher Gruppen zu verbessern. In diesem Szenario entstehen durch Synergien zwischen Kommune und Bürgerinnen und Bürgern bessere Leistungen und vielfach bessere öffentliche Wirkungen. So haben viele Kommunen Koordinationsstellen und Strukturen aufgebaut, um dem demografischen Wandel Rechnung zu tragen. In Schwäbisch Gmünd hat das Amt für Familie und Soziales ein Seniorennetzwerk $^{15}$ für ein selbstbestimmtes Leben entwickelt, das aus mehreren Bausteinen besteht. Im Mittelpunkt steht die Koordinationsstelle, die von einer qualifizierten Fachkraft geleitet wird und die Arbeit mit qualifizierten Ehrenamtlichen und freien Trägern koordiniert. Beispielsweise werden im Rahmen des Bausteins "Morgenohr“ alleinstehende ältere Menschen von Ehrenamtlichen regelmäßig telefonisch kontaktiert. Das gibt den Angerufenen nicht nur die Sicherheit, dass sich jemand um ihr Wohl kümmert, sondern auch einen wichtigen sozialen Kontakt. Insofern entsteht durch additive Koproduktion mit qualifizierten ehrenamtlichen Bürgerinnen und Bürgern ein neues kommunales Angebot, das die Lebensqualität von Senioren verbessert.

In Szenario 6 investiert die Kommune (zumindest kurzfristig) mehr Ressourcen, um durch Verhaltensänderungen die Lebensqualität bestimmter Zielgruppen mittelfristig zu verbessern. In diesem Fall leistet die Kommune als ermöglichende Verwaltung Hilfe zur Selbsthilfe, d.h. Bürger/ innen, insbesondere Nutzer/innen, werden in die Lage versetzt und motiviert, selbst Lösungen zu erarbeiten und umzusetzen. Dadurch werden öffentliche Wirkungen nachhaltig verbessert und die Kommune spart zumindest mittelfristig Ressourcen.

In Augsburg unterstützen Sozialpateninnen und Sozialpaten Menschen in finanziellen und persönlichen Notlagen.

13 Vgl. Landkreis Surrey 2010, S. 6.

14 Vgl. Tisdall 2014.

15 Löffler/Timm-Arnold/Knipp 2016, S. 25.

16 Vgl. Klopf/Stahl-Kanditt/Krell/Löffler 2016.

17 Vgl. Bundesministerium für Familie, Senioren, Frauen und Jugend (Hrsg.) 2006, S. 57. 
Familienpatenschaften richten sich an solche Familien, denen keine Partner, Freunde oder Familienangehörige zur Verfügung stehen. Oberstes Ziel der Sozial- und Familienpatenschaft ${ }^{16}$ ist die Hilfe zur Selbsthilfe: die begleiteten Menschen werden darin unterstützt, eigene Ressourcen zu erkennen, sie zu aktivieren und damit Krisen besser zu meistern. Die Studie „Lokale Bündnisse - Chancen für Akteure und Regionen“ des Prognos-Instituts im Auftrag des Bundesministeriums für $\mathrm{Fa}$ milie, Senioren, Frauen und Jugend aus dem Jahr 2006 belegt, dass die KostenNutzen-Relation des Augsburger Patenmodells deutlich positiv ist: Der Wohlfahrtsgewinn für die Kommune beläuft sich bei den Familien- und Sozialpaten auf

\section{"Effektive Formen von Koproduktion sind nicht voraussetzungslos: Es bedarf konsequenter Wirkungsorientierung und systematischer Umsetzung von Koproduktion sowie Leadership in Politik und Verwaltung."}

rund 174.000 Euro im Sinne einer Äquivalenzkostenanalyse. Dem Nutzen stehen Kosten für die Bündnisinitiative, die Begleitung und Betreuung der Freiwilligen in Höhe von 48.000 Euro gegenüber ${ }^{17}$.

\section{Nachhaltige Haushaltssicherungs- effekte durch öffentliche Wirkungsverbesserungen}

Die dargestellten Szenarien zeigen, dass sowohl durch substitutive als auch additive Formen von Koproduktion im kommunalen Haushalt Einsparungen erzielt werden können. Im Falle von substitutiven Formen von Koproduktion können bereits kurzfristig Einspareffekte erzielt werden. Im Falle additiver Formen von Koproduktion handelt es sich eher um mittelfristige Einspareffekte, da kurzfristig zunächst mehr kommunale Ressourcen investiert werden müssen. Insbeson- dere die Szenarien 2, 3 und 6 zeigen, dass durch eine wirkungsorientierte Koproduktion mit Bürgerinnen und Bürgern der kommunale Haushalt nachhaltig gesichert werden kann.

Die Dynamik und die Haushaltssicherungseffekte in Szenarien 2, 3, 5 und 6 sind jedoch unterschiedlich:

- Im Falle der substitutiven Koproduktion in den Szenarien 2 und 3 entstehen zumindest kurzfristig Einspareffekte, da das kommunale Angebot mit weniger Mitteln bereitgestellt werden kann. Wie nachhaltig diese Einspareffekte sind, hängt davon $\mathrm{ab}$, ob sich durch den Ressourceneinsatz von Bürgerinnen und Bürgern auch die Qualität
Mittel aufwenden muss, um Menschen zu Verhaltensänderungen zu motivieren und sie dabei zu begleiten.

- Aber auch additive Formen von Koproduktion, die zu keinen budgetwirksamen Einsparungseffekten führen, wie dies in Szenario 5 der Fall ist, sind unter Haushaltssicherungsaspekten zu rechtfertigen, wenn der gesamtgesellschaftliche Nutzen die Kosten übersteigt.

Derartige effektive Formen von Koproduktion sind nicht voraussetzungslos. Es bedarf konsequenter Wirkungsorientierung und systematischer Umsetzung von Koproduktion. Ein weiterer Erfolgsfaktor sind Leadership in der Politik und Verwaltung, um neue Sichtweisen und Formen der Zusammenarbeit mit Bürgerinnen und Bürgern zu institutionalisieren. Dem Bürgermeister kommt dabei eine besondere Rolle als Vermittler zwischen Lokalpolitik, Verwaltung, Bürgerschaft und örtlicher Wirtschaft zu. Dass auch die Ratspolitik bereit sein kann, ihr vermeintliches „Königsrecht“, das ist das Budgetrecht, zumindest teilweise (die abschließende Budgetentscheidung bleibt beim Rat) an die Gemeindebürger zu delegieren und damit einen breiten Konsens in der Politik und der Stadtgesellschaft mit messbaren Effekten in der Haushaltskonsolidierung zu erzielen, zeigt die folgende Fallstudie aus der niederländischen Stadt Zeist.

\section{Fallstudie: Wie Bürgerinnen und Bürgern in der niederländischen Stadt Zeist neue Lösungen zu Haushaltseinsparungen mit-ent- wickelten}

Wie überall in den Niederlanden stand auch die 60.000 Einwohner zählende Stadt Zeist in der Provinz Utrecht 2010 vor großen Haushaltsproblemen. ${ }^{19}$ Schätzungen gingen davon aus, dass mehr als zehn Prozent des beinflussbaren Budgets bzw. 6,2 Millionen Euro eingespart werden mussten. Ursprünglich bestand die Verwaltung, die Hilfe zur Selbsthilfe anbietet. Ziel ist die Verhaltensänderung wichtiger Zielgruppen (z.B. gesellschaftliche Integration vereinsamter älterer Menschen) bzw. der breiten Bürgerschaft (z.B. effizientes Energiewirtschaften). Dadurch verringert sich mittel- und langfristig die Nachfrage nach kommunalen Leistungen, wenngleich die Kommune kurzfristig mehr
18 Das Konzept political efficacy bezieht sich auf die subjektive Überzeugung von Personen, Handlungsmöglichkeiten zur Verfügung zu haben und politische Sachverhalte verstehen zu können. Vgl. Bovaird, T. et al. 2013.

19 Vgl. Schepers/Brok 2015. 
Idee darin, „beratende Bürger“ mit Szenarien und Vorschlägen zu befassen, die von der Verwaltung ausgearbeitet worden sind. Der Verwaltungsvorstand entschied sich allerdings für einen anderen, innovativeren Weg. Weil Verwaltungsprobleme letzten Endes immer auch gesellschaftliche Probleme widerspiegeln, müssen Bürgerinnen und Bürger neue Lösungen selbst erarbeiten. 200 Expertinnen und Experten aus der Bürgerschaft, die durch öffentlichen Aufruf in der örtlichen Presse und auf der Homepage der Stadt ermittelt wurden, arbeiteten in der Folge mit Verwaltungsmitarbeitern $^{20}$ in mehreren
Die vorgeschlagenen Maßnahmen summierten sich am Ende auf 7,6 Millionen Euro. Da die Deckungslücke lediglich 6,2 Mio. Euro betrug, wurde so dem Rat Gelegenheit zur eigenen politischen Prioritätensetzung gegeben. In der abschließenden Ratsdiskussion wurden 95 Prozent der Vorschläge akzeptiert; nur 15 Ratsmitglieder stimmten gegen die Sparmaßnahmen.

Die Erfahrungen aus Zeist zeigen, dass ein strategisch verankertes Mit-Entwickeln von Lösungen durch Bürgerinnen und Bürger neue Erkenntnisse für Rat und Verwaltung bringen kann, wenn diejeni-

\section{"Wenn Koproduktion nicht nur von einzelnen Innovatoren in der öffentlichen Verwaltung gefördert, sondern strategisch aufgesetzt wird, leisten substitutive und insbesondere additive Formen der Koproduktion einen wichtigen Beitrag zur nachhaltigen Haushaltssicherung."}

zogen werden, führt dies zu verbesserten Wirkungen kommunalen Handelns; auch und gerade in Zeiten knapper Kassen.

Der Fall Zeist grenzt sich auch positiv von den üblichen Verfahren im sogenannten Bürgerhaushalt ab, bei denen es sich in der Regel um rein konsultative Verfahren handelt.

\section{Ausblick}

Die lebensweltorientierte Zusammenarbeit mit sog. Bedarfs- und Leistungsträgern im Sinne von Mit-Steuern, Mit-Entwickeln, Mit-Umsetzen und Mit-Bewerten öffentlicher Wirkungen (Koproduktion), ist in vielen deutschen Kommunen und Wohlfahrtsverbänden noch Neuland. Wenn Koproduktion nicht nur von einzelnen Innovatoren in der öffentlichen Verwaltung aus Überzeugung gefördert, sondern von der Politik und Verwaltungsspitze strategisch aufgesetzt wird, leisten substitutive und insbesondere additive Formen der Koproduktion nicht nur einen wichtigen Beitrag zur Verbesserung der Lebensqualität vor Ort, sondern auch einen wichtigen Beitrag zur nachhaltigen Haushaltssicherung.

gen gezielt mit einbezogen werden, deren Alltagsexperten-Wissen und unmittelbare Erfahrungen als Nutzerinnen und Nutzer von kommunalen Dienstleistungen sonst oft im Dunkeln bleiben. Aber auch Verwaltungsmitarbeiter können durch das Wissen der Bürgerinnen und Bürger für ihre Praxis lernen. Sie können auch die "Zwänge“ und Verfahrenswege vermitteln, die die Bürgerinnen und Bürger in der Regel nicht kennen und die bedacht werden müssen. Die Fallstudie Zeist zeigt, wenn Bürgerinnen und Bürger mit ihrem Erfahrungswissen und ihren Ideen einbe-

schiedenen Gebäuden zusammen. Im Sinne der hier entwickelten Terminologie kann insofern von einer additiven Koproduktion gesprochen werden.

Mitarbeiter je Expertenkommission verantwortlich gemacht (und entsprechend geschult) für die Steuerung des Prozesses und dafür, dass die Dinge vorangingen. Die Kosten für den Beteiligungsprozess beliefen sich auf 100.000 Euro. Diese setzten sich aus den Personalkosten der eingesetzten 16 Verwaltungsmitarbeiter und den Kosten für die Versammlungsräume in ver-
21 Öffentliche Infrastruktur, Grünflächen und öffentlicher Nahverkehr; Genehmigungen; Kultur und Tourismus; Bildung; Sport und Erholung; Raumplanung; Öffentliche Sicherheit; Gesundheit und Wohlfahrt.

\section{Literatur}

Bogumil, J./Holtkamp, L. 2010: Die kommunale Ebene, in: Olk, Th./Klein, A./Hartnuß, B. (Hrsg.): Engagementpolitik. Die Entwicklung der Zivilgesellschaft als politische Aufgabe, Wiesbaden, S. 382-403

Brown, P./Löffler, E. und Christie, J. 2016: Present: Co-producing improved wellbeing for people living with dementia in East Dunbartonshire, http://www.govint.org/fileadmin/user_upload/publications/PRESENT_REPORT_2016.pdf (Abruf am 28.6.2016).

Bovaird, T. et al. 2013: Correlates of co-production: Evidence from a Five-Nation Survey of Citizens", International Public Management Journal: Vol. 16 (1), S. 1-28.

Bundesministerium für Familie, Senioren, Frauen und Jugend (Hrsg.) 2006: Die Initiative „Lokale Bündnisse für Familie" aus ökonomischer Sicht. Berlin (in Zusammenarbeit mit dem Prognos Institut), S. 57.

Klopf/Stahl-Kanditt/Krell/Löffler,E. 2016: Die Zusammenarbeit mit Sozial- und Familienpaten in Augsburg: Ein Win-Win für Paten, Nutzer und die Kommune, Governance International Fallstudie http://www.govint.org/good-practice/case-studies/ (Abruf 30.5.2016). 
Landkreis Surrey 2010: One in Ten. A needs assessment of young people aged thirteen to nineteen in Surrey. http://www.surreyyc.org. uk/NeedsAssesmentOneInTen.pdf (Abruf am 30.05.2016).

Löffler, E./Watt, P. 2009: Understanding the Efficiency Implications of Co-Production, LARCI Co-Production Research Papers, http:// www.rcuk.ac.uk/research/xrcprogrammes/ OtherProgs/larci/ (Abruf am 30.5.2016).

Löffler, E./Timm-Arnold, P./Knipp, R. 2016: Koproduktion mit Bürgern in der MitmachStadt, Innovative Verwaltung, Heft 7-8, S. 22-25.

Löffler, E./Timm-Arnold, P./Overmans, T. 2015: Wirkungsorientierte Ausgabensteuerung und Leistungsbeschaffung mit Jugendlichen im Jugendamt des Landkreises Surrey in Großbritannien, in: Hellmann, T./Löffler, E. (Hrsg.): Was machen europäische Kommunen anders? Neue Impulse für Kommunen in Deutschland, Gütersloh: Bertelsmann Stiftung, S. 20-27.

Löffler, E./Timm-Arnold, P./Bovaird, T./Van Ryzin, G. 2015: Koproduktion in Deutschland. Studie zur aktuellen Lage und den Potenzialen einer partnerschaftlichen Zusammenarbeit zwischen Kommunen und Bürgerinnen und Bürgern. Gütersloh: Bertelsmann Stiftung.

Löffler, E. 2015: Koproduktion mit Bürgerinnen, Bürgern und gemeinnützige ÖffentlichPrivate Partnerschaften: Ein transsektoraler Ansatz für eine wirkungsorientierte Verwaltungswissenschaft, Verwaltung und Management, Heft 6/2015, S. 317-324.

Löffler, E./Martin, S. 2016: Citizen engagement, in: Bovaird, T./Löffler, E. (Hrsg.): Public Management and Governance, 3rd edition, Routledge, Oxon and New York, S. 301-318.

Löffler, E. 2016: Haushaltssicherung durch Koproduktion mit BürgerInnen: Besser oder einfach nur billiger?, in Böhmer/Kegelmann/ Kientz, Rechnungswesen und Controlling. Das Steuerungshandbuch für Kommunen, Heft 7 oder 8 (im Erscheinen).

Schepers, A./Brok, E. 2015: Zeist (NL): Spardialog mit Bürgerinnen und Bürgern, in: Hellmann, T./Löffler, E. (Hrsg.): Was machen europäische Kommunen anders? Neue Impulse für Kommunen in Deutschland. Gütersloh: Bertelsmann Stiftung, S. 6-11.

Timm-Arnold, P./Löffler, E. 2013: Vom Vater Staat zum Partner Staat: Bürgerlnnen als Koproduzenten öffentlicher Leistungen und Wirkungen, Verwaltung und Management, Heft 4/2013, S. $197-204$.

Tisdall, C. 2014: The Transformation of services for young people in Surrey County Council, http://www.govint.org/good-practice/case-studies/the-transformation-of-services-for-youngpeople-in-surrey-county-council/ (Abruf am 30.06.2016).

Vogel, H.-J. 2016: Neue Form von Koproduktion öffentlicher Leistungen: „Neue Nachbarn Arnsberg“ oder „Flüchtlinge“ arbeiten mit an neuen Lösungen der Stadtentwicklung, http:// www.arnsberg.de/buergermeister/veroeffentlichungen/2016/neue-nachbarn.pdf (Abruf am 31.05.2016).

\section{Frische Gesetze}

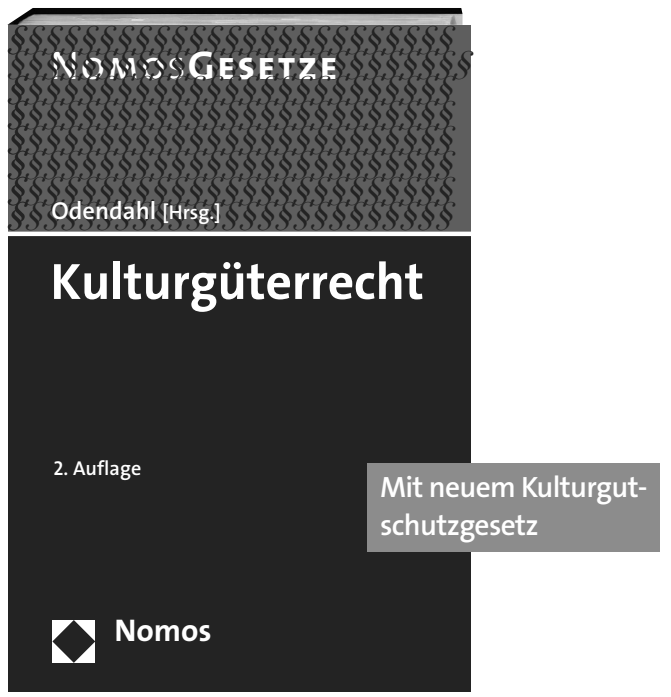

\section{Kulturgüterrecht}

Herausgegeben von Prof. Dr. Kerstin Odendahl

2. Auflage 2017, ca. 960 S., brosch., ca. 58,- $€$

ISBN 978-3-8487-3647-8

Erscheint ca. Februar 2017

nomos-shop.de/28635

Das Werk umfasst als einzige vollständige Sammlung alle für die Bundesrepublik Deutschland geltenden kulturgüterschützenden Normen auf völker-, europa-, bundes- und landesrechtlicher Ebene. Berücksichtigt sind Normen zum Erhalt von Kulturgütern, zur Verhinderung ihrer illegalen Ausfuhr und zur Kulturförderung.

\section{Stimmen zur Vorauflage:}

\Da es die erste Sammlung dieser Art in Deutschland ist, ist es zugleich die Visitenkarte der Bundesrepublik Deutschland, soweit nach der Geltung der Kulturgüter- und Denkmalschutzvorschriften in Deutschland gefragt wird... Somit kommt niemand, der sich für Kulturgüterschutz interessiert, künftig an diesen ausgezeichneten Pionierleistungen vorbei. Ernst-Rainer Hönes, DSI 1/06

Die Normensammlung von Kerstin Odendahl ist für Praktiker und Wissenschaftler eine reichhaltige Erkenntnisquelle. Sie ist ein weiterer Beitrag zum zwischenzeitlich entstandenen sonderrecht Kulturgüterrecht.

Mark Pawlytta, Frankfurt a. M., KUR 3-4/07 《

Portofreie Buch-Bestellungen unter www.nomos-shop.de

Alle Preise inkl. Mehrwertsteuer

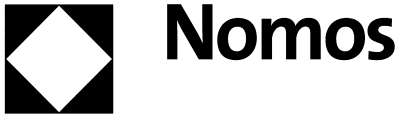

